\title{
Preface
}

\section{Engineering Turbulence Modelling, Simulation and Measurements}

\author{
Michael Leschziner ${ }^{1}$ - Mike Reeks ${ }^{2}$ - Wolfgang Rodi $^{3}$. \\ Luc Vervisch ${ }^{4}$
}

This Special Issue contains substantially extended and revised versions of 18 papers, selected from a total of 159 contributions presented at the three-days Symposium Engineering Turbulence Modelling and Measurements-10 (ETMM-10), held in Marbella, Spain, in September 2014, under the auspices of ERCOFTAC. The ETMM series of events was established to give greater prominence and sharper profile to research on applications-oriented aspects of turbulence and its computational and experimental characterisation, thus helping to bridge the gap between fundamental approaches to turbulence and the exploitation of models, codes and knowledge in an industrial setting. In this respect, ETMM is thematically closely consonant with the aims and ethos of Flow, Turbulence and Combustion (FTaC).

Within the broad spectrum of topics featuring at ETMM meetings, the majority deal with computational and experimental approaches to complex aero- and hydro-dynamic flows, multi-phase and reacting flows, flow control, flows in power generation and environmental fluid mechanics. The present Special Issue aspires to reflect this mix and to provide an archival record of some of the best papers presented at ETMM-10. To this end, selected papers were subjected to the same rigorous review process as that followed in respect of any other contribution to FTaC, each manuscript being reviewed by two (in many cases, three) leading experts, with some manuscripts undergoing two revisions before being accepted.

The 18 papers included in this Special Issue report research in seven major areas: flow control - with drag reduction, separation control, transition suppression and plasma actuation being focal points; transition - on a rotatng disc, on a sphere and on curved surfaces;

Michael Leschziner

mike.leschziner@imperial.ac.uk

1 Imperial College London, London, UK

2 Newcastle University, Newcastle upon Tyne, UK

3 Karlsruhe Institute of Technology (KIT), Karlsruhe, Germany

4 INSA de Rouen, Saint-Étienne-du-Rouvray, France 
reacting flows - flame extinction, flame unsteadiness around bluff bodies, fuel-lean combustion and novel analyses of DNS data to support combustion modelling being the main issues; modelling and/or simulation of boundary layers, jets and complex recirculating flows; experimental techniques based on novel PIV/PTV for complex flows; particle transport and deposition; and a review of current DNS capabilities in aero-acoustics.

The Editors hope that the readers of this Special Issue will judge it to be a valuable contribution to the literature, and to give credit to efforts by the scientific community to engage in turbulence modelling, simulation and measurements of immediate relevance to the industrial arena.

Michael Leschziner, Special Issue Guest Editor

Mike Reeks

Wolfgang Rodi

Luc Vervisch 